\title{
Neonatal blood glucose concentrations: metabolic effects of intravenous glucagon and intragastric medium chain triglyceride
}

\author{
J M Hawdon, A Aynsley-Green, M P Ward Platt
}

\begin{abstract}
Neonatal hypoglycaemia is a common clinical problem and the traditional treatment for the condition is intravenous glucose administration. The glycaemic effects of two additional treatments were investigated, in a randomised trial, in 23 neonates who were receiving intravenous glucose as treatment for hypoglycaemia. Eleven infants received an intravenous bolus dose of glucagon $(200 \mu \mathrm{g} / \mathrm{kg})$ and 12 infants received intragastric medium chain triglyceride (MCT) (5 $\mathrm{ml} / \mathrm{kg})$. Blood concentrations of glucose and intermediary metabolites were measured before and one hour after treatment, and, using stable isotope infusion, glucose production rates were calculated.
\end{abstract}

After glucagon, there was a mean rise in blood glucose concentration of $1.6 \mathrm{mmol} / \mathrm{h}$ and in the glucose production rate of 2.6 $\mathrm{mg} / \mathrm{kg} / \mathrm{min}$. After MCT, there was a small mean rise in blood glucose concentration of $0.4 \mathrm{mmol} / \mathrm{h}$, but the effect of MCT on glucose production rate was variable.

Intravenous bolus glucagon administration exerted a glycaemic effect which was associated with an increase in glucose production rate. The use of glucagon, as an alternative treatment to intravenous glucose, should be investigated further.

\section{(Arch Dis Child 1993;68:255-261)}

Although the definition and significance of neonatal hypoglycaemia remains controversial, ${ }^{1}$ the prevention and management of the condition almost invariably involves intravenous glucose administration, especially when enteral feeding is poorly tolerated. However, for the mature infant who could otherwise be managed on the postnatal ward, this has the disadvantages of separating mother and baby, creating parental anxiety, interfering with the establishment of milk feeding, and increasing the neonatal nursery workload. In addition, rebound hypoglycaemia may occur on discontinuing the infusion, and this form of treatment does not address the underlying failure of metabolic adaptation in these infants, of which hypoglycaemia is only one aspect. ${ }^{2}$

The hyperglycaemic response to glucagon was described over 40 years ago by Cornblath et $a l,{ }^{3}$ and subsequent studies have suggested that its mechanism of action is via cAMP induction of phosphenolpyruvate carboxyki- nase, the major enzyme of gluconeogenesis, resulting in increased endogenous glucose production. ${ }^{4-6}$ Glucagon also enhances glycogenolysis and ketogenesis.

Enteral administration of medium chain triglyceride (MCT) has also been shown to have a glycaemic effect in babies who were not enterally fed, but its use as an adjunct to enteral milk feeding has not been described. It has been suggested that the mechanism of action is by stimulating ketogenesis, which, in turn, promotes gluconeogenesis by the provision of energy to the gluconeogenic pathway. ${ }^{7} 8$ In addition, the provision of ketone bodies may exert a glucose sparing effect by acting as alternative fuels.

Our aim was to investigate the glycaemic effects of these two therapies which, if effective, could supplement or replace intravenous glucose treatment, either as prophylaxis or as treatment for neonatal hypoglycaemia.

\section{Subjects and methods}

The subjects were 23 infants who had persistent hypoglycaemia, that is, at least two laboratory (Yellow Springs Instrument) blood glucose measurements $<2.6 \mathrm{mmol} / \mathrm{l}$ on the day of study, and who were to receive treatment for the hypoglycaemia, a decision made by the attending medical staff. The aim of the study was to determine the blood metabolite concentrations and the glucose production rates for hypoglycaemic neonates, before and after treatment with glucagon or MCT. However, current management of persistent neonatal hypoglycaemia consists of intravenous glucose infusion, at rates of at least $5 \mathrm{mg} / \mathrm{kg} / \mathrm{min}$. It was considered unethical to delay or alter this management in infants recruited to the study.

In fact, for 10 of the 23 infants, the attending medical staff had felt it necessary to commence intravenous glucose infusions before the start of the study, so that, for these babies, it has not been possible for the investigator (JMH) to confirm the diagnosis, or to obtain blood samples for metabolite and hormone concentrations, when the babies were hypoglycaemic. These infants were already receiving intravenous glucose (at rates of at least 5 $\mathrm{mg} / \mathrm{kg} / \mathrm{min}$ ) at the start of the study, and, for these infants, the glucose infusion rate was not altered during the course of the study. At the start of the study (time 0 ), blood was collected from these infants for the subsequent assay of circulating concentrations of glucose, intermediary metabolites, insulin, and glucagon. 
For the other 13 infants, who were not already receiving intravenous glucose, before the commencement of intravenous glucose infusion (time 0 ), at a rate of $5 \mathrm{mg}$ glucose/ $\mathrm{kg} / \mathrm{min}$, blood samples were collected for the subsequent measurement of blood glucose and intermediary metabolite concentrations and the plasma concentrations of insulin and glucagon (see below). For these infants, the glycaemic effect of the glucose infusion alone could be determined, before investigating the glycaemic effects of glucagon or MCT.

For all infants, determination of glucose production rates, as described below, could be performed only after the commencement of intravenous glucose infusion which had corrected the hypoglycaemia in all but one of the subjects. No infant received a bolus of intravenous glucose.

All 23 infants received, simultaneously with the prescribed glucose infusion, a primed, constant infusion of 6,6 dideuterated glucose at a rate of $120 \mu \mathrm{g} / \mathrm{kg} / \mathrm{min}$. After one hour of infusion, three heelprick blood samples, of 0.5 $\mathrm{ml}$ each, were taken at 15 minute intervals (times $60,75,90 \mathrm{~min}$ ), for the subsequent assay of blood concentrations of glucose and intermediary metabolites, plasma insulin and glucagon concentrations, and plasma isotope enrichment. Eleven babies were then randomised to receive a bolus intravenous dose of glucagon $(200 \mu \mathrm{g} / \mathrm{kg})$ and 12 to receive a single intragastric dose of MCT emulsion (5 $\mathrm{ml} / \mathrm{kg}$ by gavage). One hour after administration, two further heelprick samples were collected for metabolite and isotope enrichment assays (times 150, $165 \mathrm{~min}$ ).

A6 it was not possible to calculate the contribution of enteral milk feeds to the total glucose appearance rate, enteral feeds were withheld from all the infants during the study, that is from the time of commencement of stable isotope infusion. Therefore, glucese production rates were determined at least one hour after the last milk feed.

Blood intermediary metabolite concentrations were determined by microenzymatic assays using an autoanalyser, and plasma insulin and glucagon concentrations by radioimmunoassay, as described previously. ${ }^{9}$ Plasma isotope enrichment was assayed by gas chromatography-mass spectrometry, and glucose production rate calculated as described previously. ${ }^{9}$ Isotopic steady state was assumed if the SD of the three isotope enrichment values before treatment was less than $10 \%$ of the mean of the three values. Similarly, for the two samples taken one hour after treatment, steady state was assumed if the difference between the two values was less than $10 \%$ of the mean of the two values.

A birthweight SD score was calculated for each infant, that is the number of SDs by which the birth weight differed from the mean for gestational age.

Plasma insulin and non-esterified fatty acid (NEFA) concentrations, and blood ketone body concentrations had log normal distributions, so $\log _{10}$ values were used in statistical analysis. For these data, ranges, rather than
$95 \%$ confidence intervals are given in the tables, and the means given are geometric means. The values for lactate, pyruvate, alanine, and glycerol were summed to give the total gluconeogenic substrate concentration. For the three samples taken during isotope infusion, before treatment, the mean values for glucose and metabolite concentrations were used for analysis. Baseline information for the groups of infants were compared by $t$ test, the response to treatment in each group was assessed by paired $t$ test, and intergroup differences in the response to treatment by $t$ test. Pearson correlation coefficients were used to describe relationships between variables.

Ethical approval was granted by the ethics committee of Newcastle Health Authority, and parents gave informed consent for the inclusion of their baby in the study.

\section{Results}

The characteristics of the 13 infants who were sampled before glucose infusion are shown in table 1. Some of these infants were found to have high plasma insulin at low blood glucose concentrations (fig 1). During the glucose infusion, the mean blood glucose concentration rose significantly (table 2 ), but the mean plasma insulin concentrations were unchanged (fig 1). For infants with high baseline insulin, there was no increase in insulin concentration at the higher glucose concentration during infusion.

Presumed underlying causes of hypoglycaemia for all 23 infants, divided into groups according to the treatment they subsequently

Table 1 Details of the 13 infants who were sampled before and after intravenous glucose infusion

\begin{tabular}{lll}
\hline & Median & Range \\
\hline Gestation (weeks) & 37 & $33-40$ \\
Birth weight (g) & 2080 & $1610-3480$ \\
Birthweight SD score & $-2 \cdot 27$ & $-3 \cdot 33$ to $0 \cdot 78$ \\
MAC:HC* & $0 \cdot 26$ & $0 \cdot 21-0 \cdot 32$ \\
Postnatal age (hours) & 24 & 12 to 340 \\
Diagnosis: & 10 & \\
$\quad$ Small for gestational age & 1 & \\
$\quad$ Maternal gestational diabetes & 1 & \\
$\quad$ Unknown & 2 & \\
\hline
\end{tabular}

*MAC: $\mathrm{HC}=$ mid-arm circumference:head circumference ratio.

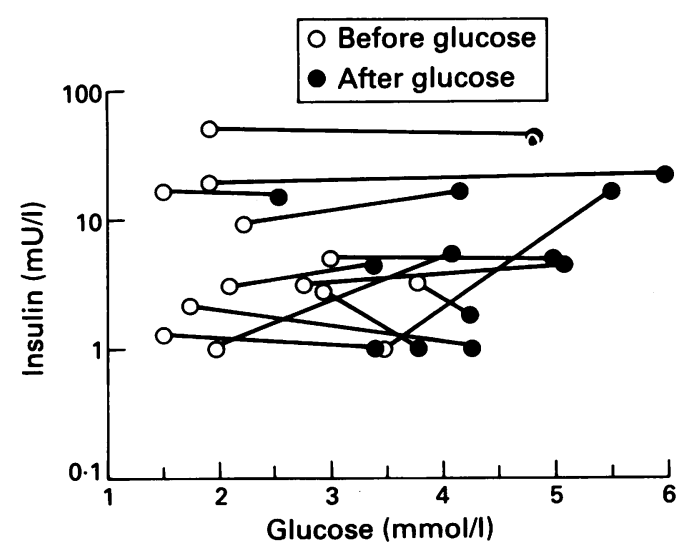

Figure 1 The effect of intravenous glucose ( $5 \mathrm{mg} / \mathrm{kg} / \mathrm{min})$ on blood glucose and plasma insulin concentrations. 
Table 2 Mean metabolite and hormone concentrations before and during intravenous glucose treatment and mean ratio for values during treatment:before treatment (95\% confidence intervals)

\begin{tabular}{|c|c|c|c|}
\hline & $\begin{array}{l}\text { Before treatment } \\
(n=13)\end{array}$ & $\begin{array}{l}\text { During treatment } \\
(n=13)\end{array}$ & $\begin{array}{l}\text { During:before treatment } \\
(n=13)\end{array}$ \\
\hline $\begin{array}{l}\text { Glucose }(\mathrm{mmol} / \mathrm{l}) \\
\text { Total gluconeogenic substrate }(\mathrm{mmol} / \mathrm{l}) \\
\text { Ketone body }(\mathrm{mmol} / \mathrm{l}) \\
\text { Glucagon }(\mathrm{pmol} / \mathrm{l}) \\
\text { Insulin (mU/l) } \\
\text { Insulin:glucagon ratio }\end{array}$ & $\begin{array}{l}2.40(2.00 \text { to } 2 \cdot 78) \\
3.25(2.66 \text { to } 3 \cdot 84) \\
0.07(0.04 \text { to } 0 \cdot 11) \\
43.30(34 \cdot 80 \text { to } 51 \cdot 70) \\
4.40(2 \cdot 20 \text { to } 8 \cdot 60) \\
0.11(0.05 \text { to } 0.21)\end{array}$ & $\begin{array}{c}4.30(3.80 \text { to } 4.90) \\
3.00(2.55 \text { to } 3.46) \\
0.05(0.04 \text { to } 0.08) \\
42.50(29.8 \text { to } 55 \cdot 20) \\
5.50(2.70 \text { to } 11.90) \\
0.14(0.07 \text { to } 0.32)\end{array}$ & $\begin{array}{l}1.9(1.6 \text { to } 2.2)^{*} \\
0.9(0.8 \text { to } 1.1) \\
1.1(0.6 \text { to } 1.6) \\
0.9(0.4 \text { to } 1.5) \\
2.5(0.2 \text { to } 4.8) \\
3.0(0.0 \text { to } 6.0)\end{array}$ \\
\hline
\end{tabular}

Paired $t$ test, before $v$ during treatment: ${ }^{\star} \mathrm{p}<0 \cdot 001$.

Table 3 Diagnoses according to treatment group

\begin{tabular}{lll}
\hline & $\begin{array}{l}\text { Glucagon } \\
(n=11)\end{array}$ & $\begin{array}{l}\text { MCT } \\
(n=12)\end{array}$ \\
\hline Small for gestational age (<10th centile) & 6 & 11 \\
Unknown & 4 & - \\
Maternal gestational diabetes & 1 & - \\
Perinatal asphyxia & - & 1 \\
\hline
\end{tabular}

received, in addition to intravenous glucose, are shown in table 3, and details of the infants and baseline metabolic data in table 4. By chance, there were a number of differences between the groups: the infants in the MCT treatment group were smaller for gestational age and had lower pretreatment glucagon concentrations and higher pretreatment glucose production rates, although the ranges were very wide for each group and glucose concentrations were similar between groups.

Glucose turnover values were available for eight infants in each treatment group, with steady state (as defined above) being achieved after one hour of infusion. The babies in the glucagon treatment group had low glucose production rates, while there was a wide range of glucose production rates for the MCT group. For all infants, there was no significant correlation of glucose production rate with insulin or glucagon concentration or with insulin:glucagon ratio.

After glucagon treatment (figs 2, 3) there were significant increases in blood total gluconeogenic substrate and glucose concentrations $(\mathrm{p}<0.05, \mathrm{p}<0.001$, respectively), significant decreases in blood ketone body and plasma NEFA concentrations $(p<0.01, p<0.05$, respectively), and a significant increase in glucose production rate $(p<0 \cdot 01)$. However, there was no direct relationship between the increase in glucose production rate and the increase in blood glucose concentration.

After MCT treatment (figs 2, 3) there were small, but significant, increments in mean blood glucose and ketone body concentrations $(\mathrm{p}<0.05, \mathrm{p}<0.01$, respectively). Where the initial glucose production rates were low, there was an increased rate after MCT treatment, while rates fell after treatment in those babies who had high initial glucose production rates. In the MCT treatment group, there were no relationships between the increase in ketone body concentration, the change in glucose concentration or the change in glucose production rate.

The changes after treatment are compared in table 5. The mean increases in total gluconeogenic substrate and glucose concentrations and glucose production rate were more significant after glucagon than after MCT, but MCT resulted in more significant increases in NEFA and ketone body concentrations.

\section{Discussion}

This study was an observational study, the subjects of which were a group of infants who had aroused clinical concern, before the study, because of persistent hypoglycaemia. The infants were managed according to current unit policies; that is, by optimising enteral milk intake, and then commencing intravenous glucose treatment if milk feeding alone did not prevent hypoglycaemia. The investigators did not alter this management, but examined the effects of two additional treatments that may, in the future, supplement enteral milk feeding, and perhaps replace the need for intravenous glucose

Table 4 Details of infants, and baseline metabolic data, according to treeatment group; results are mean (95\% confidence intervals) unless otherwise stated

\begin{tabular}{|c|c|c|c|c|}
\hline \multirow[b]{2}{*}{ Gestation (weeks) } & \multicolumn{2}{|c|}{$\begin{array}{l}\text { Glucagon } \\
(n=11)\end{array}$} & \multicolumn{2}{|c|}{$\begin{array}{l}M C T \\
(n=12)\end{array}$} \\
\hline & $36 \cdot 3$ & 34.9 to $37 \cdot 7)$ & $36 \cdot 3$ & $(34 \cdot 7$ to $37 \cdot 8)$ \\
\hline Birth weight $(\mathrm{g})$ & 2322 & (2000 to 2644 ) & 1947 & (1681 to 2213 ) \\
\hline Birthweight SD score & $-1 \cdot 45$ & $(-2 \cdot 17$ to -0.73$)$ & $-2 \cdot 50$ & $(-2.96$ to $-2 \cdot 04) *$ \\
\hline MAC: $\mathrm{HC}+$ & 0.27 & $(0.26$ to 0.28$)$ & $0 \cdot 25$ & $(0.24$ to 0.26$)$ \\
\hline Median (range) postnatal age (hours) & 24 & (6 to 340$)$ & 37 & $(19$ to 312$)$ \\
\hline Feed volume ( $\mathrm{ml} / \mathrm{kg} /$ day $)$ & 105 & ( 85 to 130$)$ & 110 & (88 to 132$)$ \\
\hline Median (range) time since last feed (min) & 110 & $(60$ to 240$)$ & 90 & (60 to 240$)$ \\
\hline Intravenous glucose $(\mathrm{mg} / \mathrm{kg} / \mathrm{min})$ & $4 \cdot 7$ & $(4 \cdot 1$ to $5 \cdot 3)$ & $5 \cdot 1$ & $(3.6$ to 6.6$)$ \\
\hline Glucose $(\mathrm{mmol} / \mathrm{l})$ & $4 \cdot 1$ & $(3.3$ to 4.8$)$ & $3 \cdot 5$ & $(2 \cdot 8$ to $4 \cdot 2)$ \\
\hline Total gluconeogenic substrate $(\mathrm{mmol} / \mathrm{l})$ & 3.06 & $(2 \cdot 24$ to 3.87$)$ & $2 \cdot 53$ & $(1.92$ to 3.13$)$ \\
\hline Ketone body $(\mathrm{mmol} / \mathrm{l})$ & 0.05 & $(0.03$ to 0.07$)$ & 0.04 & $(0.02$ to 0.06$)$ \\
\hline $\mathrm{NEFA}(\mathrm{mmol} / \mathrm{l})$ & 0.15 & $(0.12$ to 0.41$)$ & $0 \cdot 13$ & $(0.06$ to 0.29$)$ \\
\hline Insulin (mU/l) & $4 \cdot 4$ & $(2 \cdot 3$ to $8 \cdot 4)$ & $4 \cdot 1$ & $(1.9$ to 8.9$)$ \\
\hline Insulin:glucagon ratio & $0 \cdot 10$ & $(0.05$ to 0.18$)$ & $0 \cdot 16$ & $(0.06$ to 0.39$)$ \\
\hline Glucagon $(\mathrm{pmol} / \mathrm{l})$ & $53 \cdot 5$ & $(33.6$ to 73.4$)$ & $29 \cdot 6$ & $(22 \cdot 3 \text { to } 36 \cdot 9)^{*}$ \\
\hline Glucose production rate $(\mathrm{mg} / \mathrm{kg} / \mathrm{min})$ & $1 \cdot 6$ & $(0.9$ to 2.3$)$ & $5 \cdot 2$ & $(2 \cdot 8$ to $7 \cdot 6) *$ \\
\hline
\end{tabular}

†MAC: $\mathrm{HC}=$ mid-arm circumference head circumference ratio

By $t$ test, glucagon $v$ MCT: * $p<005$. 


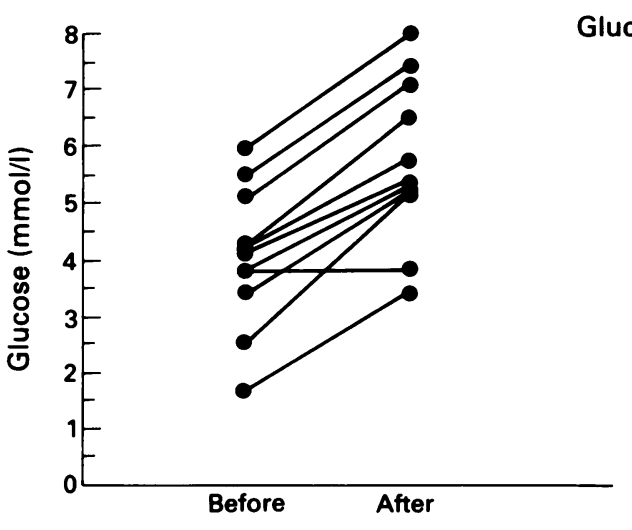

Glucagon
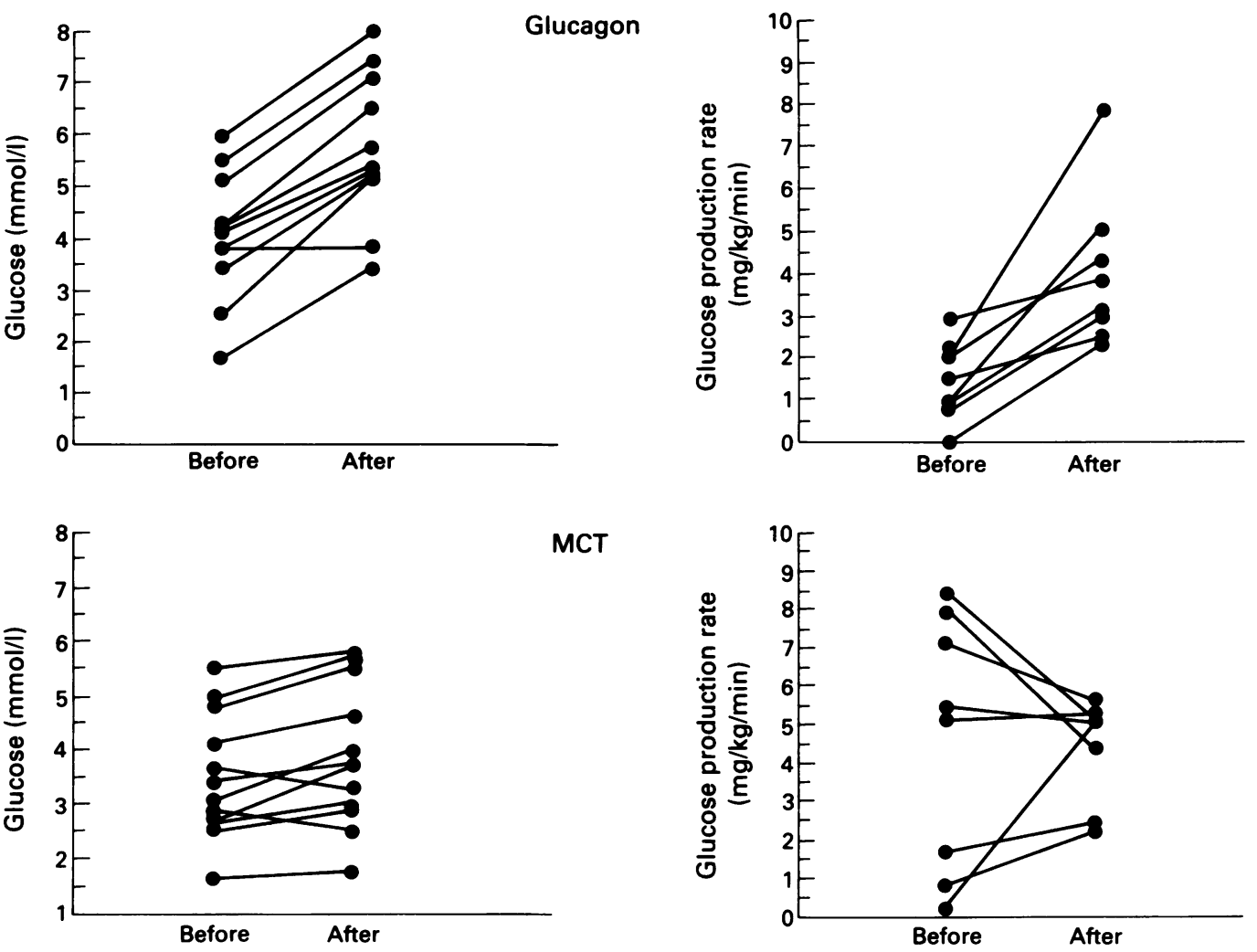

MCT

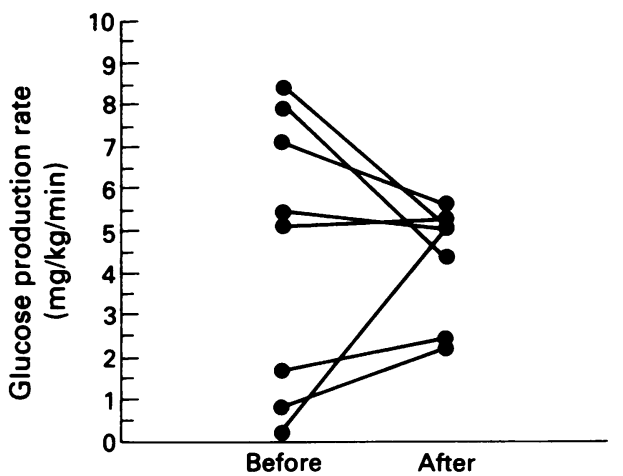

Figure 2 Changes in blood glucose concentrations and glucose production rates after glucagon and MCT treatment.
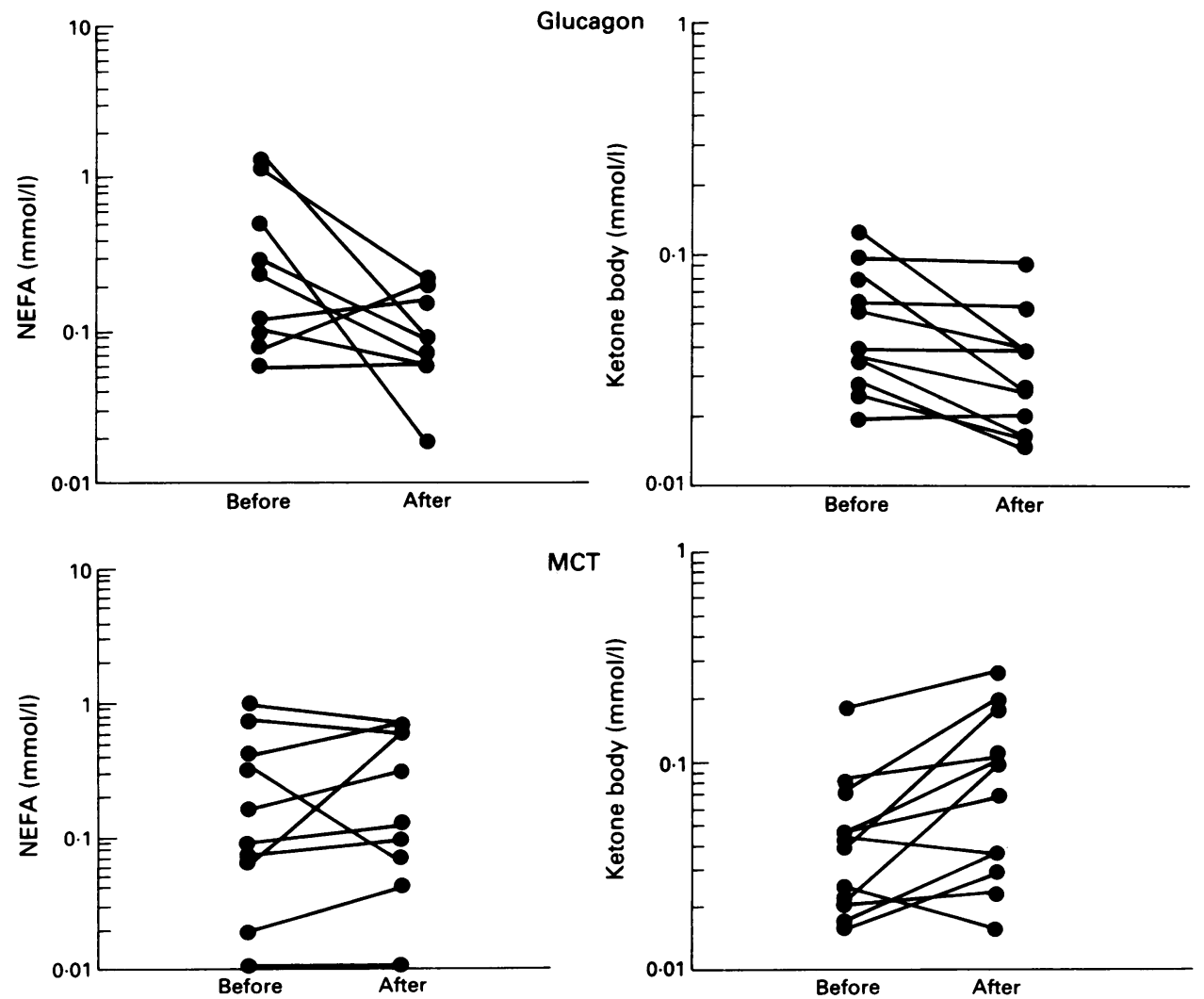

Figure 3 Changes in plasma NEFA and blood ketone body concentrations after glucagon and MCT treatment.

treatment. All of the infants in the present study appeared to have hypoglycaemia despite substantial enteral milk intakes (table 4). The clinical decision to treat some of these infants with intravenous glucose before the study precluded the confirmation of the clinical diagnosis of hypoglycaemia, using our own glucose assay.

Ethical considerations prevented the investigation of glucose turnover rates in hypoglycaemic infants who were not treated with intravenous glucose. Therefore, we were 
Table 5 Comparison of mean before treatment:after treatment ratios (95\% confidence intervals) for metabolic values

\begin{tabular}{|c|c|c|}
\hline & $\begin{array}{l}\text { Glucagon } \\
(n=11)\end{array}$ & $\begin{array}{l}M C T \\
(n=12)\end{array}$ \\
\hline Glucose $(\mathrm{mmol} / \mathrm{l})$ & $1.5(1.3$ to 1.6$)$ & $(1 \cdot 0 \text { to } 1 \cdot 2)^{* * *}$ \\
\hline Total gluconeogenic substrate $(\mathrm{mmol} / \mathrm{l})$ & $1.3(1.1$ to 1.4$)$ & $(0.9 \text { to } 1 \cdot 1)^{*}$ \\
\hline Ketone body $(\mathrm{mmol} / \mathrm{l})$ & $0.9(0.8$ to 1.0$)$ & $(1 \cdot 1 \text { to } 1 \cdot 3)^{* * *}$ \\
\hline $\mathrm{NEFA}(\mathrm{mmol} / \mathrm{l})$ & $0.8 \quad(0.7$ to 1.0$)$ & $(1.0 \text { to } 1 \cdot 2)^{*}$ \\
\hline Glucose production rate $(\mathrm{mg} / \mathrm{kg} / \mathrm{min})$ & $2.9 \quad(2.0$ to 3.8$)$ & $4 \cdot 3(0 \text { to } 10 \cdot 3)^{*}$ \\
\hline
\end{tabular}

By $t$ test, glucagon $v$ MCT: ${ }^{*} \mathrm{p}<0 \cdot 05 ; * * \mathrm{p}<0 \cdot 001$.

unable to investigate the effect of the two treatments on infants who were receiving enteral feeds alone, in the absence of intravenous glucose. An additional factor preventing this comparison was the inability to calculate the contribution to glucose turnover rates made by enteral milk feeds. However, by measuring glucose production rates, before and after treatment with glucagon or MCT, we were able to compare the effects of each treatment with that of intravenous glucose alone, each infant acting as his or her own control. Therefore, there was no requirement to study a further group of infants who received neither treatment. Future studies should investigate the actions of each treatment in enterally fed infants by measuring changes in blood glucose concentrations.

The same ethical considerations precluded the measurement of glucose production rates at the time of hypoglycaemia. For those infants with low glucose production rates during the study, the intravenous glucose infusion and correction of hypoglycaemia may have suppressed endogenous glucose production. Alternatively, there may have been little change in glucose production rate once the intravenous glucose infusion was commenced, and, if this were so, hypoglycaemia may have been secondary to low glucose production rates. Most of the infants had endogenous glucose production rates less than normal basal value, of $5 \mathrm{mg} / \mathrm{kg} / \mathrm{min}$, reported by other workers. ${ }^{10}$ However, the values in the present study were similar to those previously found for glucose infused infants. ${ }^{11}$

Three infants in the glucagon group and four infants in the MCT group failed to achieve steady state. Data regarding changes in blood glucose concentration, but not glucose production rates, were available for these infants. The absence of turnover data for these infants may have introduced a bias into the comparison between the groups. For example, it is possible that these infants had either an upward or a downward trend in endogenous glucose production rate before the treatment was given, which would either amplify or mask the apparent effect of the treatment. However, in these seven infants, there was little variation in each subject's blood glucose concentration during either the period before or after treatment. This suggests that any increase in blood glucose concentration seen after treatment was a true treatment effect.

In terms of treatment of hypoglycaemia, this study has demonstrated, first, that intravenous glucose treatment, at a constant infusion rate of $5 \mathrm{mg} / \mathrm{kg} / \mathrm{min}$, adequately corrected hypogly- caemia, without causing a disproportionate increase in plasma insulin concentrations, even in those infants with high basal insulin concentrations. This supports the findings of a recent study of hyperglycaemic babies, which demonstrated a poor insulin secretory response to hyperglycaemia, but a close relationship between blood glucose concentration and intravenous glucose infusion rate (unpublished data). It appears that neonatal glucose homoeostasis is more dependent on external influences (such as glucose infusion rate) than upon internal control mechanisms.

Second, we have shown that a single intravenous injection of glucagon had a marked glycaemic effect in infants who had been hypoglycaemic, and that this effect was present irrespective of the blood glucose concentration at the time of treatment. Also, glucagon treatment was associated with a small increase in gluconeogenic substrate concentration and an increased endogenous glucose production rate. These findings suggest that glucagon stimulated both the release of gluconeogenic precursors and their utilisation in gluconeogenesis, and that this was the likely mechanism of the glycaemic action of glucagon. Gluconeogenic substrate turnover studies would yield further information about the contribution of gluconeongenesis to the glycaemic effect of glucagon. We were unable to assess the contribution of glycogenolysis to the increased glucose production rate, but as the majority of these infants were at least 12 hours old, and were small for gestational age, it is likely that their diminished glycogen stores had already been utilised. ${ }^{12}$

The absence of a direct relationship between the increases in both glucose production rate and blood glucose concentration after glucagon may reflect the timing of sampling, one hour after treatment. The acute changes in glucose production rate may have occurred soon after administration, after which production rates and concentrations settled to a new steady state. The methods involved in calculating glucose turnover rate, in that constant isotope infusion over one hour must occur to achieve steady state, necessitate such a lag period.

Previous studies have examined the metabolic effects of glucagon infusion, but did not measure glucose turnover rates. ${ }^{13} 14$ Others, which have described a glycaemic effect of bolus glucagon administration, of a similar order to that found in the present study, also failed to measure glucose production rates. ${ }^{41516}$ The only other study, to date, which recorded glucose production rates after bolus glucagon administration, was of single infants and did not have standardised treatment or study conditions. $^{6}$ Therefore, this study is the first to demonstrate the mechanism of the glycaemic effect of bolus glucagon administration, by measuring the concentrations of glucose and intermediary metabolites and the glucose turnover rates of a group of infants under controlled conditions.

Third, we have demonstrated that intragastric MCT administration was associated with small but significant increases in blood glucose and 
ketone body concentrations. The increases in blood glucose and ketone body concentrations, and in glucose production rate, were less than those reported by Sann et al. ${ }^{8}$ However, the infants in the latter study received no enteral or parenteral energy for at least three hours before study, which resulted in low blood glucose concentrations and, possibly, maximal stimulation of ketogenesis, whereas in the present study, continued glucose infusion led to higher pretreatment blood glucose and may have partially suppressed ketogenesis.

There are two possible mechanisms of action of MCT: first, the provision of fatty acid substrates may have promoted $\beta$ oxidation and thus released cofactors and energy for gluconeogenesis, or, second, ketone body production may have had a glucose sparing effect, by providing alternative fuels to glucose. In the first instance, an increase in glucose production rate would be anticipated, in the second instance the glucose sparing effect of alternative fuels would allow a reduction in glucose turnover rate at steady state. Therefore our findings, of a variable glucose turnover response to MCT, may reflect these different mechanisms.

It was not possible to anticipate, before the study, the glucose turnover rates of each infant, and it is by chance that some of the infants in the MCT treatment group had high initial glucose turnover rates. None the less, it is of interest that, for these infants, MCT resulted in reduced turnover rate (a possible glucose sparing effect), while, for those with low initial turnover rates, MCT resulted in increased turnover rate (possibly by promoting gluconeogenesis). However, there were too few infants in the MCT treatment group to assess the separate mechanisms of action, and turnover studies, using stable isotopically labelled ketone bodies, would be required to investigate the effect of MCT on fatty acid metabolism. The lack of a relationship between change in glucose production rate with changes in blood glucose or ketone body concentrations is also explained by the above discussion.

Finally, when the changes associated with the two treatments were compared, glucagon appeared to be more effective in terms of increasing glucose production rate and blood glucose concentration. However, we were unable to assess in the present pilot study whether intramuscular administration of glucagon would be as effective as intravenous administration, or whether the response to treatment is so effective as to remove the need for intravenous glucose infusion altogether. Further studies are needed to assess the optimal dosage and administration regimens (doses used in previous studies range from $3 \mu \mathrm{g} / \mathrm{kg}$ to $300 \mu \mathrm{g} / \mathrm{kg}$ ). Moreover, earlier studies have demonstrated that the use of higher dose glucagon is limited by an insulinogenic effect at these doses. ${ }^{4614}$ In the present study, ethical constraints prevented our taking sufficient blood for glucoregulatory hormone assays after treatment.

Consideration of only the glycaemic effect of treatment is inadequate because newborn infants may utilise ketone bodies in preference to glucose. Glucagon treatment did not appear to have a ketogenic effect, although the increase in gluco- neogenic substrate concentrations may indicate that alternative fuels, such as lactate were made available. On the other hand, the ketogenic capacity of MCT treatment may be more valuable than its glycaemic effects. In this way, although MCT does not have a marked glycaemic effect, it may be a more rational treatment for neonatal hypoglycaemia. However, an important practical disadvantage of MCT treatment, as evident in the present study, is the variability of response, which may have been related to the efficiency of absorption after enteral administration. Therefore MCT treatment can only be used for those infants who will tolerate enteral feeds. Further studies should investigate the effects of MCT used as an adjunct to enteral milk feeds, rather than in a situation where enteral feeds are withheld, as in this study and that of Sann et al. ${ }^{8} \mathrm{~A}$ second disadvantage is the small risk that a hypoglycaemic infant may have a disorder of fatty acid $\beta$ oxidation, in which case MCT administration would exacerbate any metabolic disturbance.

This study has demonstrated that glucagon has a glycaemic effect, associated with increased glucose production rates. Therefore, it is a potential treatment for neonatal hypoglycaemia, and may reduce the intravenous glucose requirement of hypoglycaemic infants. Its use in the treatment of hypoglycaemia, without concurrent intravenous glucose infusion, should be evaluated. Finally, if such treatment is effective, prophylactically, in preventing hypoglycaemia in very small for gestational age infants (for example those with birth weight $<1$ st centile), or those who have evidence of disproportionate growth retardation (with low mid-arm circumference:head circumference ratios), it may remove the need for intravenous glucose treatment and thus reduce admission rates to neonatal special care nurseries.

Dr Hawdon was supported by the Scientific and Research Committee of Newcastle Health Authority and by a Foundation for the Study of Infant Deaths training fellowship.

We thank the parents who gave consent for the inclusion of their We thank the parents who gave consent for the inclusion of their
babies in the study. We acknowledge the assistance of the following: babies in the study. We acknowledge the assistance of the following:
Ms A McGann, Department of Child Health, University of Ms A McGann, Department of Child Health, University of
Newcastle upon Tyne, with metabolite assays; Mr C Hetherington and Dr K Bartlett, Biomedical Mass Spectrometry Unit, University of Newcastle upon Tyne with turnover studies; Dr M Ghatei and Professor S Bloom, Hammersmith Hospital, London with glucagon assays; and Ms L Ashworth and Professor KG M M Alberti, Diabetic Research Group, Department of Medicine, University of Newcastle upon Tyne, with insulin assays.

1 Comblath M, Schwartz R, Aynsley-Green A, Lloyd J. Hypoglycaemia in infancy: the need for a rational definition. Pediatrics 1990;85:834-7.

2 Hawdon JM, Ward Platt MP. Metabolic adaptation in small for gestational age infants. Arch Dis Child 1993;68:262-8.

3 Comblath M, Levin EY, Marquetti E. The effect of glucagon on the concentration of sugar in the capillary blood of the on the concentration of sugar in the

4 Linarelli LG, Bobik C, Bobik J, Drach AL, Rubin HM The effect of glucagon on adenosine-3',5'-monophosphate (cyclic AMP), glucose, insulin and growth hormone in full term newborn children. F Clin Endocrinol Metab 1974; 39:411-7.

5 Bussey ME, Finlay S, Ogata ES. Hypoglycaemia in the newborn growth retarded rat. Delayed phosphoenolpyruvate carboxykinase induction despite increased glucagon availability Pediatr Res 1985;19:363-7.

6 Mehta A, Wootton R, Cheng KL, Penfold P, Halliday D, Stacey TE. Effect of diazoxide or glucagon on hepatic glucose production rate during extreme neonatal hypoglycaemia. Arch Dis Child 1987;62:924-30.

7 Sann L, Mathieu M, Lasne Y, Ruitton A. Effect of oral administration of lipids with $67 \%$ medium chain triglycerides on glucose homeostasis in preterm neonates. Metabolism 1981; 30:712-6.

8 Sann L, Divry P, Lasne Y, Ruitton A. Effect of oral lipid administration on glucose homeostasis in small for gestation age infants. Acta Paediatr Scand 1982;71:923-7.

9 Hawdon JM, Aynsley-Green A, Bartlett K, Ward Platt MP. The role of pancreatic insulin secretion in neonatal glucore- 
gulation. II. Infants with disordered blood glucose homoeostasis. Arch Dis Child 1993;68:280-5.

10 Bougneres PF. Stable isotope tracers and the determination of fuel fluxes in newborn infants. Biol Neonate 1987;52(suppl 1):87-96.

11 Cowett RM, Susa JB, Oh W, Schwartz R. Glucose kinetics in glucose infused small for gestational age babies. Pediatr Res 1984;18:74-9.

12 Shelley HJ, Neligan GS. Neonatal hypoglycaemia. $\mathrm{Br}$ Med Bull 1966;22:34-9.

13 Schultz K, Mestyan J, Soltesz GY, Horvath M. The metabolic effects of glucagon infusion in normoglycaemic and hypoglycaemic small for gestational age infants. I Changes in blood glucose, blood lactate and plasma free fatty acids. Acta Paediatrica Academiae Scientianum Hungaricae 1976; 17:237-44

14 Carter PE, Lloyd DJ, Duffy P. The use of glucagon in the management of hypoglycaemia in the small for gestational management of hypoglycaemia in the small for gestational age infant

15 Milner RDG, Wright AD. Plasma glucose, non-esterified fatty acids, insulin and growth hormone response to glucagon in the newborn. Clin Sci 1967;32:249.

16 Le Dune MA. Response to glucagon in small for dates hypoglycaemic and non-hypoglycaemic newborn infants. Arch Dis Child 1972;47:754-9. 